\title{
Terahertz Time-Domain Spectroscopy
}

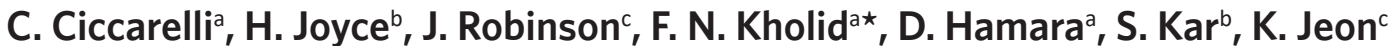

Time-Domain terahertz spectroscopy (THz TDS) has attracted attention from many scientific disciplines as it enables accessing the gap between electronic and optical techniques. One application is to probe spintronic dynamics in sub-picosecond time scale. Here, we discuss principles and technical aspects of a typical THz TDS setup. We also show an example of terahertz time-domain data obtained from a Co/Pt thin film calibrant, which is a well-studied spintronic structure emitting strong $\mathrm{THz}$ radiation. See video at https://youtu.be/X7vrvQcmy8c

\section{Introduction}

The new THz spectroscopy laboratory at the Maxwell Centre (Cavendish Laboratory, University of Cambridge) became fully operational in March 2019 (Figure 1). The laboratory was mainly funded by The Winton Programme for the Physics of Sustainability through an Advanced Research Fellowship awarded to Chiara Ciccarelli (Figure 2), in combination with funding from the Royal Society and the European Union, belonging to both Chiara Ciccarelli and Hannah Joyce.

Time-domain THz Spectroscopy, is a powerful technique for materials characterisation and control. ${ }^{1-4}$ Technologies to generate $\mathrm{THz}$ radiation are now widely available and this is what is allowing a wider use of $\mathrm{THz}$ frequencies in new research areas. One of these areas is spintronics. 5,6

Spintronics means 'spin transport electronics' and indicates electronics made of spins as opposed to electronics made of charges. 5,6 The spin is a fundamental property of electrons, which makes them behave as very small compass needles. The spin of an electron has a direction and can point up or down or in between. Spin currents can be used to transfer information even in insulators, where charge transport is suppressed. ${ }^{7.8}$ Spintronics has enabled higher-density and cheaper hard-drive storage via breakthrough technologies such as the giant magnetoresistance. ${ }^{9-12}$ The combination of magnetic tunnelling junctions and spin-transfer-torque allowed the fabrication of a new generation of magnetic random-access memories. In a spin-transfer-torque junction, spin-polarised charges enter the ferromagnet and can switch its magnetisation from one state to the other by generating torques. This is used to electrically write zero and one bits. ${ }^{13}$

In anti-ferromagnets the magnetic moments at crystal lattice sites, rather than pointing all in the same direction as in ferromagnets, point in alternate directions. This means that an anti-ferromagnet always has a zero net magnetisation. However its internal magnetic ordering can be used to store information. In an anti-ferromagnetic memory device the writing speed in the terahertz frequency range of a one or zero bit is a thousand times faster than in ferromagnets. ${ }^{14,15}$

Studying anti-ferromagnets is one of the reasons why we invested in developing experimental techniques that are sufficiently fast to resolve their magnetic dynamics. This is why we decided to build a $\mathrm{THz}$ spectroscopy setup in our laboratory.

\section{Time-Domain THz Spectroscopy Setup}

Our terahertz time-domain spectroscopy (THz TDS) is capable of performing $\mathrm{THz}$ transmission and $\mathrm{THz}$ emission spectroscopy. In the case of $\mathrm{THz}$ transmission, we steer a THz pulse to a sample and detect the transmitted $\mathrm{THz}$ pulse as the output signal (Figure 3).

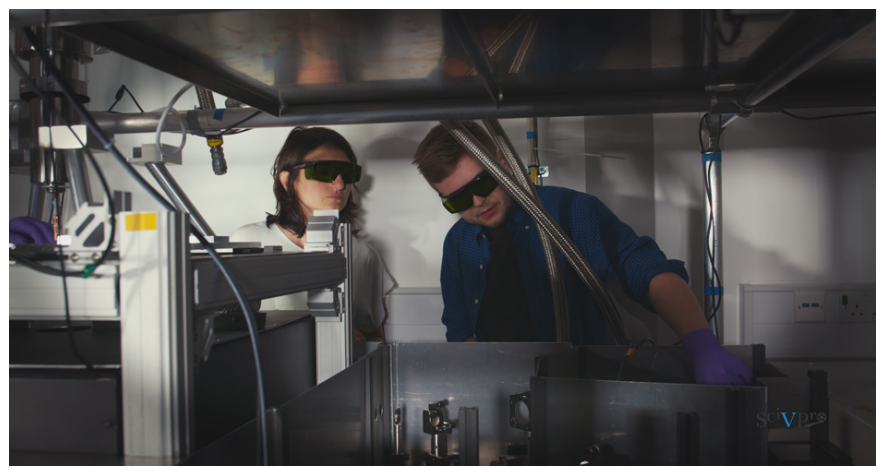

Figure 1 | Chiara Ciccarelli (Lecturer at the University of Cambridge) and Dominik Hamara (PhD student).

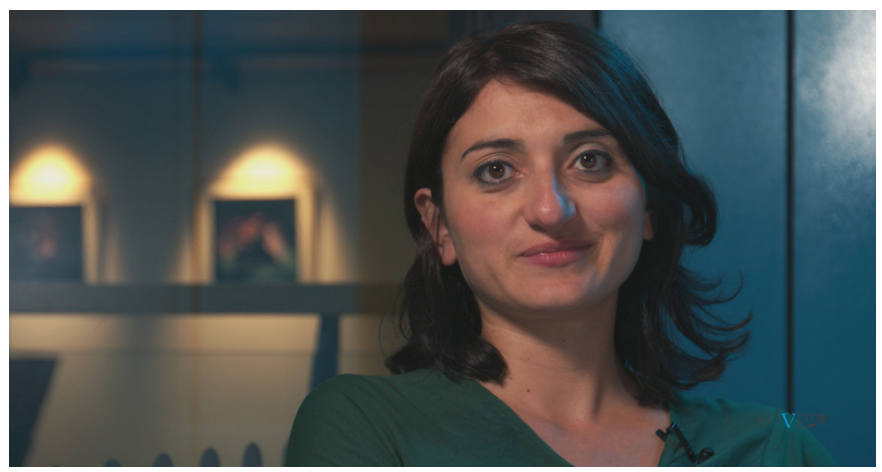

Figure 2 | Chiara Ciccarelli, University lecturer at the Maxwell Centre.

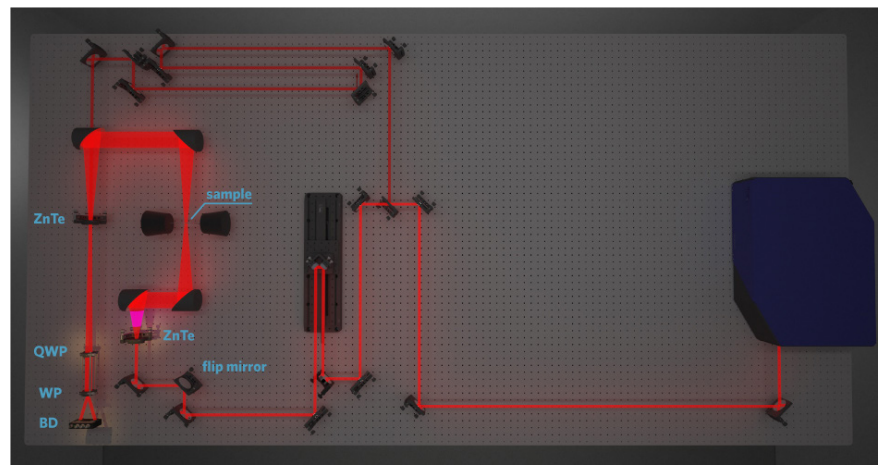

Figure 3 | Time-Domain THz Spectroscopy transmission mode configuration overview. The flip mirror is used to switch between modes.

a*Cavendish Laboratory, University of Cambridge, Cambridge, UK, (fnk23@cam.ac.uk), b Department of Engineering, University of Cambridge, Cambridge, UK, ${ }^{c}$ Department of Materials Science and Metallurgy, University of Cambridge, Cambridge, UK. 
To do this, we have a zinc-telluride crystal which emits $\mathrm{THz}$ when being pumped by a femtosecond optical pulse.

Whereas for the THz emission, the optical pulse hits the sample directly (Figure 4). The output signal is a $\mathrm{THz}$ pulse emitted by the sample itself.

For both transmission and emission measurements, the output $\mathrm{THz}$ signal lasts several picoseconds. Because standard optical detectors do not have a picosecond resolution, a type of pump-probe detection technique is used to measure the output signal with a sub-picosecond resolution. The technique is called electro-optic sampling, where an electro-optic crystal is hit by the output $\mathrm{THz}$ signal as the pump, and a weak optical pulse as the probe. By measuring the change in the probe pulse transmitting through the crystal, we can construct the output $\mathrm{THz}$ signal in the time-domain.

In this setup a Ti:sapphire amplified laser (Solstice Ace, Spectra-Physics) produces $40 \mathrm{fs}$ optical pulses at a central infrared wavelength of $800 \mathrm{~nm}$. A great fraction of the laser power is used to generate the $\mathrm{THz}$ pulse while the remaining fraction is used for detecting the $\mathrm{THz}$ signal. The source wavelength can also be changed with an optical parametric amplifier (Topas-Prime \& NirUVis, Light Conversion).

The femtosecond laser beam bounces off a series of mirrors and passes through standard alignment optics before reaching a beam splitter. Here, $99 \%$ of the transmitted beam is used as a pump-beam exciting the sample, and the other $1 \%$ is used as a probe-beam for time-domain detection.

The pump-beam travels towards a delay line, which is used to introduce a relative time delay between the pump and the probe pulses. Different delays allow sampling different data points from the $\mathrm{THz}$ signal exiting the sample; meaning that the $\mathrm{THz}$ output signal can be reconstructed by sweeping the delay line. After being delayed, the pump can take two different paths.

In the $\mathrm{THz}$ emission mode, the pump hits the sample directly (Figure 4). The black box encapsulates the sample and the part of the optical setup where the $\mathrm{THz}$ radiation propagates (Figure 5). Because water absorbs and reemits at the $\mathrm{THz}$ frequency range, spurious signals can be prevented by filling the box with nitrogen gas. The beam is then collimated by a concave lens into a chopper and passes through a parabolic mirror.

The sample is attached on a copper sample holder and mounted on a cryostat, which can be used to control the sample temperature between 3 and 500K (Figure 6, DE-210SF, Advanced Research Systems, USA). A magnetic field up to $1 \mathrm{~T}$ can be applied to the sample with an electromagnet (5403, GMW).

In transmission mode (Figure 3), the infrared pump-beam goes through a ZnTe non-linear crystal (Greyhawk Optics ZnTe-10-10$1-\mathrm{H})$ to generate a $\mathrm{THz}$ beam that is then transmitted through the sample. ${ }^{16,17}$ Here, the beam size is adjusted to fit a chopper wheel and then focussed before entering a black box.

The zinc-telluride crystal emits a $\mathrm{THz}$ pulse due to a $2^{\text {nd }}$ order non-linear electro-optical process called optical rectification, where bound charges respond asymmetrically to the incoming optical wave. ${ }^{16}$ These bound charges respond differently to different direction of the perturbing electric field giving rise to a non-zero electric polarization averaged over one oscillation period. In the case of a continuous wave, the resulting electric polarization is constant over time. However, for a femtosecond pulse, its intensity changes dramatically over the envelope of the pulse. Hence, electric dipoles change rapidly and emit radiations that are in the $\mathrm{THz}$ band. Ideally, the emitted THz pulse should have a bandwidth of 1 over the optical pulse width (e.g. 100fs results in a $10 \mathrm{THz}$ bandwidth). However, the non-linear crystal properties impose some limitations, such as $\mathrm{THz}$ absorption dips ${ }^{18}$ and phase matching condition between the optical pulse and $\mathrm{THz}$ pulse, ${ }^{18,19}$ which make the emitted $\mathrm{THz}$ bandwidth narrower. The $1 \mathrm{~mm}$ thick ZnTe employed in our setup, for example, has a frequency band that extends from $0.1 \mathrm{THz}$ to $\sim 2.5-3 \mathrm{THz} .{ }^{18-20}$

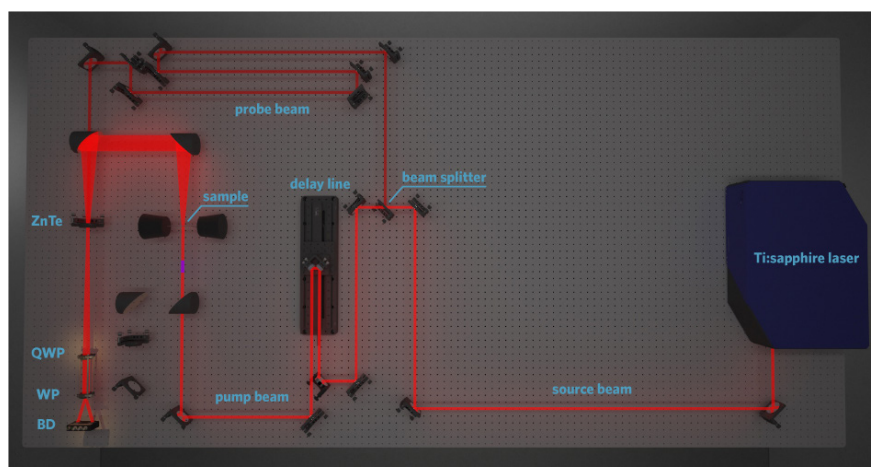

Figure 4 | Time-domain $\mathrm{THz}$ spectroscopy emission mode configuration overview. QWP, WP, and BD indicate a quarter wave plate, a Wollaston prism, and a balanced photodiode.

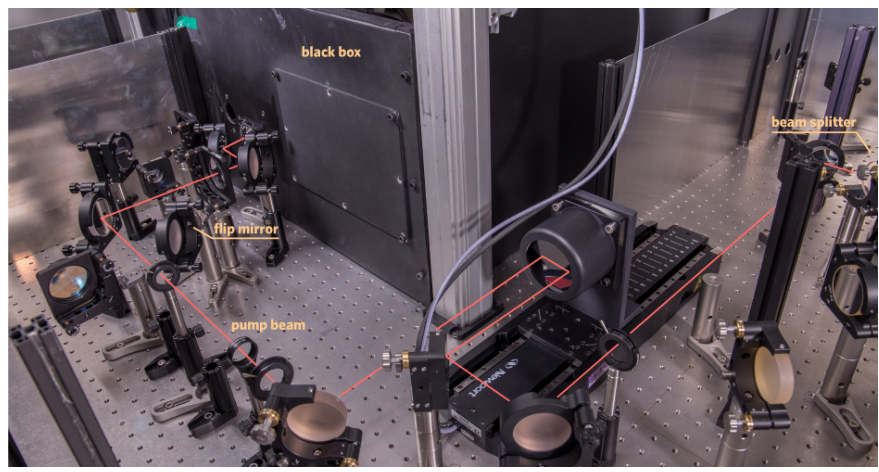

Figure 5 | Time-Domain THz Spectroscopy emission mode configuration.

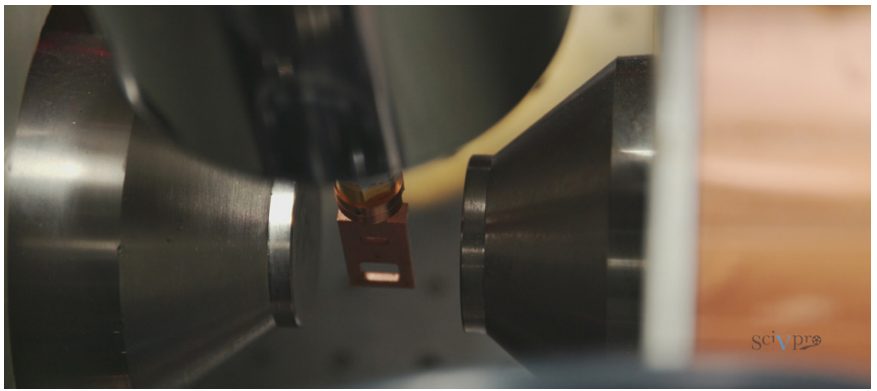

Figure 6 | Sample holder mounted on the cryostat and aligned to the electromagnet.

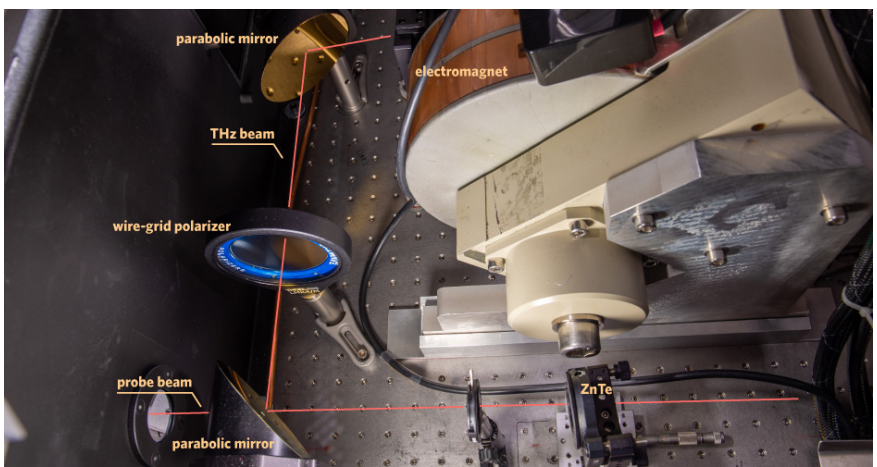

Figure 7 | Time-Domain THz Spectroscopy inside the black box.

Other crystals, such as GaP or organic crystals, ${ }^{21-23}$ are characterized by a broader bandwidth or giving a more intense $\mathrm{THz}$ output and can be used instead of $\mathrm{ZnTe}$ depending on the setup requirements.

This THz pulse is then focussed to the sample by two parabolic mirrors. 
The rest of the setup is the same for both configurations.

The probe-beam is steered such that its path length roughly matches the pump path length and the time-resolved detection can take place, as discussed later.

The probe-beam enters the black box and goes through a parabolic mirror (Figure 7). The $\mathrm{THz}$ beam coming from the sample passes through a wire-grid polariser mounted on a motorized stage, reflected by a parabolic mirror, and goes through another wire-grid polariser, before reaching a second parabolic mirror.

The $\mathrm{THz}$ and probe-beams meet at this parabolic mirror and travel together through the black box. The THz and the probe-beams spatially and temporally overlap at a zinc-telluride crystal (Figure 4, Figure 3, Figure 7), where the electro-optic sampling occurs. The probe pulse passes through a quarter-wave plate (QWP, AQWP05M-980, Thorlabs) and through a Wollaston prism (WP, WP10-B, Thorlabs), where the probe is split into its orthogonal components, before reaching a balanced photodiode (BD, PDB201A, Thorlabs).

Before running an actual measurement, the detection optical assembly needs to go through a calibration procedure without any $\mathrm{THz}$ signal. A probe pulse exiting the crystal without an appropriate phase would result in a non-zero detector output even without any signal. The compensation procedure consists in adjusting the quarter-wave plate until the reading of the balanced photodiode is close to zero volts, or, equivalently, until the probe-pulse exiting the quarter-wave plate is circularly polarised. Once this calibration has been carried out, the THz pulse can be measured. The THz-induced birefringence modifies the electric field of the probe from circular to elliptical. This deviation, which is proportional to the $\mathrm{THz}$ field, is detected by the balanced photodiode. By probing the photodiode output as a function of the delay between the THz-pulse and the probe-pulse, the $\mathrm{THz}$ electric field can be reconstructed in the time-domain. ${ }^{24}$ A lock-in amplifier is used with the chopper shown earlier to improve the signal to noise ratio of the reconstructed signal.

\section{Measurements Instructions}

In this section we illustrate how to run a calibration measurement in emission mode with the $\mathrm{THz}$ time-domain spectroscopy setup (see https://youtu.be/X7vrvQcmy8c for details).

1. Make sure the setup is configured in emission mode.

2. Mount the sample on the sample holder.

3. Mount the sample holder on the cryostat.

4. Use the pendant crane control to bring down the sample (Figure 8) until it is centred between the two electromagnetic poles. Use extra care to avoid collisions with setup components.

5. Turn on the electromagnet power supply. The electromagnet is used to align the magnetisation of the sample. Slowly increase the current up to 20 Ampere.

6. Wear safety googles, switch the titanium-sapphire laser power supply on and turn the three safety keys clockwise. Activate the laser from the laser control software.

7. To start a new measurement select 'Standard Multiple Iterations' from the THz time-domain spectroscopy control software, click on 'Measurement Setting Enable', and press 'Start'.

8. The probe monitor represents the probe pulse used for the electro-optical sampling. The probe must be stable, with a maximum deviation of $1 \%$ from the baseline.

\section{Results}

Here we perform a calibration measurement of $\mathrm{Co} / \mathrm{Pt}$ thin film bilayer in emission mode after magnetising the Co layer with the electromagnet. ${ }^{25-27}$

The Co/Pt sample is supposed to emit a broadband signal up to $30 \mathrm{THz}$, but here the FFT is only up to $2 \mathrm{THz}$. This is due to the cut-off frequency of the zinc-telluride crystal in the detection area.
At low frequencies the sensitivity is limited by $\mathrm{THz}$ diffraction as our beam spot size is comparable with the $\mathrm{THz}$ wavelength in this range. ${ }^{18,19}$ Figure 9 and Figure 10 show a comparison between the acquired data and the reference data as reported by Li et al., in the time-domain and frequency domain, respectively. ${ }^{28}$

The good match between measurements suggests that the setup is well configured and ready for an actual measurement.

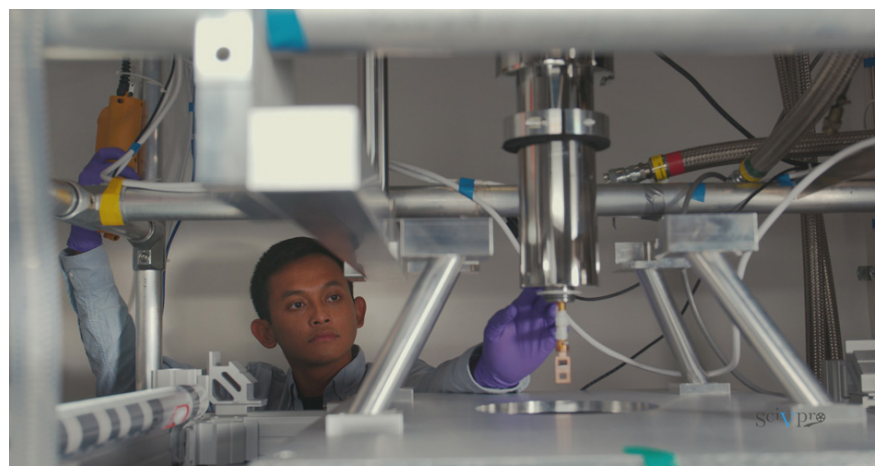

Figure 8 | Farhan Nur Kholid (PhD Student) demonstrating the Time-domain $\mathrm{THz}$ spectroscopy setup and measurement.

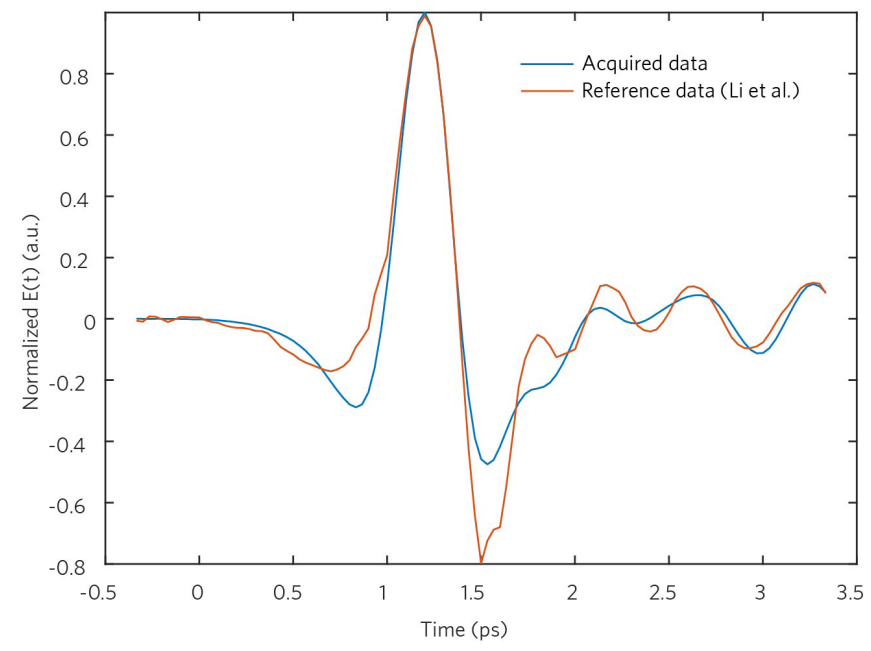

Figure 9 | Time-domain data of terahertz emission from Co/Pt thin film bilayer, acquired data vs reference data (Li et al, figure $3 \mathrm{~b}$ ). ${ }^{28}$

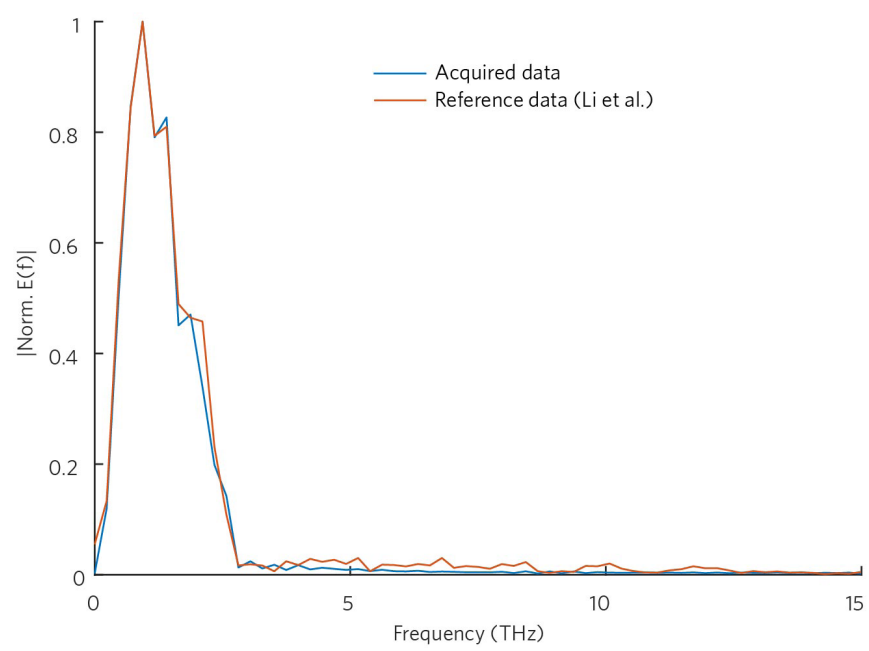

Figure 10 | Frequency-domain data of terahertz emission from Co/Pt thin film bilayer, acquired data vs reference data (Li et al, figure $3 b){ }^{28}$ 


\section{References}

1 M. Dressel, N. Drichko, B. Gorshunov \& A. Pimenov. THz spectroscopy of superconductors. IEEE Journal on Selected Topics in Quantum Electronics 14, 399-406, (2008).

2 R. A. Kaindl, M. A. Carnahan, J. Orenstein, D. S. Chemla, H. M. Christen, H.Y. Zhai, M. Paranthaman \& D. H. Lowndes. Far-Infrared Optical Conductivity Gap in Superconducting MgB2 Films. Physical Review Letters 88, (2002).

3 M. Beck, M. Klammer, S. Lang, P. Leiderer, V. V. Kabanov, G. N. Gol'Tsman \& J. Demsar. Energy-gap dynamics of superconducting NbN thin films studied by time-resolved terahertz spectroscopy. Physical Review Letters 107, (2011).

4 P. H. Siegel. Terahertz technology. IEEE Transactions on Microwave Theory and Techniques 50, 910-928, (2002).

5 A. Fert. Origin, Development, and Future of Spintronics (Nobel Lecture). Angewandte Chemie International Edition 47, 5956-5967, (2008).

6 S. A. Wolf, D. D. Awschalom, R. A. Buhrman, J. M. Daughton, S. von Molnár, M. L. Roukes, A. Y. Chtchelkanova \& D. M. Treger. Spintronics: A Spin-Based Electronics Vision for the Future. Science 294, 1488, (2001).

7 P. Gambardella \& I. M. Miron. Current-induced spin-orbit torques. Philosophical Transactions of the Royal Society A: Mathematical, Physical and Engineering Sciences 369, 3175-3197, (2011).

8 A. Brataas, A. D. Kent \& H. Ohno. Current-induced torques in magnetic materials. Nature Materials 11, 372-381, (2012).

9 M. N. Baibich, J. M. Broto, A. Fert, F. N. Van Dau, F. Petroff, P. Etienne, G Creuzet, A. Friederich \& J. Chazelas. Giant Magnetoresistance of (001) Fe/(001)Cr Magnetic Superlattices. Physical Review Letters 61, 2472-2475, (1988).

10 S. Bhatti, R. Sbiaa, A. Hirohata, H. Ohno, S. Fukami \& S. N. Piramanayagam. Spintronics based random access memory: a review. Materials Today 20, 530548, (2017).

11 K. L. Wang, J. G. Alzate \& P. Khalili Amiri. Low-power non-volatile spintronic memory: STT-RAM and beyond. Journal of Physics D: Applied Physics 46, 074003, (2013).

12 G. Binasch, P. Grünberg, F. Saurenbach \& W. Zinn. Enhanced magnetoresistance in layered magnetic structures with antiferromagnetic interlayer exchange. Physical Review B 39, 4828-4830, (1989).

13 C. Chappert, A. Fert \& F. N. Van Dau. The emergence of spin electronics in data storage. Nature Materials 6, 813-823, (2007).

14 C. Marrows. Addressing an antiferromagnetic memory. Science 351, 558, (2016).

15 T. Jungwirth, X. Marti, P. Wadley \& J. Wunderlich. Vol. 11 231-241 (Nature Publishing Group, 2016).

16 M. Bass, P. A. Franken, F. Ward \& G. Weinreich. Opical Rectification. Physical Review Letters 9, 28-31, (1962).

17 A. Rice, Y. Jin, X. F. Ma, X. C. Zhang, D. Bliss, J. Larkin \& M. Alexander. Terahertz optical rectification from $\langle 110\rangle$ zinc-blende crystals. Applied Physics Letters 64, 1324-1326, (1994).

18 A. Schneider, M. Neis, M. Stillhart, B. Ruiz, R. U. A. Khan \& P. Günter. Generation of terahertz pulses through optical rectification in organic DAST crystals: theory and experiment. Journal of the Optical Society of America B 23 , 1822-1822, (2006).

19 A. Nahata, A. S. Weling \& T. F. Heinz. A wideband coherent terahertz spectroscopy system using optical rectification and electro-optic sampling. Applied Physics Letters 69, 2321-2323, (1996).

20 A. Tomasino, A. Parisi, S. Stivala, P. Livreri, A. C. Cino, A. C. Busacca, M. Peccianti \& R. Morandotti. Wideband THz time domain spectroscopy based on optical rectification and electro-optic sampling. Scientific reports 3, 31163116, (2013)

21 C. P. Hauri, C. Ruchert, C. Vicario \& F. Ardana. Strong-field single-cycle THz pulses generated in an organic crystal. Applied Physics Letters 99, (2011).

22 C. Vicario, A. V. Ovchinnikov, S. I. Ashitkov, M. B. Agranat, V. E. Fortov \& C. P. Hauri. Generation of 09-mJ THz pulses in DSTMS pumped by a Cr:Mg 2SiO_4 laser. Optics Letters 39, 6632-6632, (2014).

23 C. Vicario, M. Jazbinsek, A. V. Ovchinnikov, O. V. Chefonov, S. I. Ashitkov, M. B. Agranat \& C. P. Hauri. High efficiency THz generation in DSTMS, DAST and $\mathrm{OH} 1$ pumped by $\mathrm{Cr}$ :forsterite laser. Optics Express 23, 4573-4573, (2015).
Distortion-free enhancement of terahertz signals measured by electro-optic sampling I Theory. Journal of the Optical Society of America B 31, 904-904, (2014).

25 T. J. Huisman, R. V. Mikhaylovskiy, J. D. Costa, F. Freimuth, E. Paz, J. Ventura, P. P. Freitas, S. Blügel, Y. Mokrousov, T. Rasing \& A. V. Kimel. Femtosecond control of electric currents at the interfaces of metallic ferromagnetic heterostructures. Nature Nanotechnology 11, 455-458, (2015).

26 T. Seifert, S. Jaiswal, U. Martens, J. Hannegan, L. Braun, P. Maldonado, F. Freimuth, A. Kronenberg, J. Henrizi, I. Radu, E. Beaurepaire, Y. Mokrousov, P. M. Oppeneer, M. Jourdan, G. Jakob, D. Turchinovich, L. M. Hayden, M. Wolf, M. Münzenberg, M. Kläui et al. Efficient metallic spintronic emitters of ultrabroadband terahertz radiation. 10, (2015).

27 H. S. Qiu, K. Kato, K. Hirota, N. Sarukura, M. Yoshimura \& M. Nakajima. Layer thickness dependence of the terahertz emission based on spin current in ferromagnetic heterostructures. Optics Express 26, 15247-15247, (2018).

28 G. Li, R. V. Mikhaylovskiy, K. A. Grishunin, J. D. Costa, T. Rasing \& A. V. Kimel. Laser induced $\mathrm{THz}$ emission from femtosecond photocurrents in $\mathrm{Co} /$ $\mathrm{ZnO} / \mathrm{Pt}$ and $\mathrm{Co} / \mathrm{Cu} / \mathrm{Pt}$ multilayers. Journal of Physics D: Applied Physics 51, 134001, (2018).

\section{Conflicts of Interest}

There are no conflicts to declare.

\section{Acknowledgements}

The authors acknowledge the funding by The Winton Programme for the Physics of Sustainability and Royal Society. H.J. acknowledges support from ERC Starting Grant (ACrossWire grant number 716471). J.R. acknowledges support from EPSRC through a Programme Grant (EP/M50807/1) and International Network (EP/ P026311/1). F.K. acknowledges support from Jardine Foundation and Cambridge Trust. The authors also thank Roger Beadle, Thomas Sharp, Chris Burling, Ollie Norris, and Gary Large for technical support.

\section{Author Contributions}

F.K. and C.C. wrote the manuscript. F.K and D.H. built the optical setup and measured the $\mathrm{Co} / \mathrm{Pt}$ bilayer. K.J and J.R fabricated the $\mathrm{Co} /$ Pt samples. H.J. and S.K. provided advice on building Terahertz setup. All authors contributed to this work.

\section{Keywords}

Terahertz, ultrafast spectroscopy, spintronics, time domain thz spectroscopy, time domain terahertz spectroscopy, thz, thz spectroscopy, spin.

\section{Article Information}

This article is licensed by C. Ciccarelli et al. under a Creative Commons Attribution 4.0 International License (ㄷ BY 4.0). 